$\mathrm{IC} / 98 / 94$

TUW-98-21

hep-th/9810038

\title{
General Solution of String Inspired Nonlinear Equations
}

\author{
I. Bandos 用 \\ The Abdus Salam ICTP, \\ P.O. Box 586, \\ 34100, Trieste, Italy \\ and \\ Institute for Theoretical Physics, \\ NSC Kharkov Institute of Physics and Technology, \\ 310108, Kharkov, Ukraine \\ E. Ivanovin \\ Bogoliubov Laboratory of Theoretical Physics, JINR, \\ 141980 Dubna, Moscow Region, Russia \\ A. A. Kapustnikov f \\ Department of Physics, Dnepropetrovsk University, \\ 320625, Dnepropetrovsk, Ukraine \\ S. A. Ulanov \\ Department of Physics, Dnepropetrovsk University, \\ 320625, Dnepropetrovsk, Ukraine
}

\begin{abstract}
We present the general solution of the system of coupled nonlinear equations describing dynamics of $D$-dimensional bosonic string in the geometric (or embedding) approach. The solution is parametrized in terms of two sets of the left- and right-moving Lorentz harmonic variables providing a special coset space realization of the product of two $(D-2)$ dimensional spheres

$$
S^{D-2}=\frac{S O(1, D-1)}{S O(1,1) \times S O(D-2) \otimes K_{D-2}} .
$$

The bosonic string (and $p$-brane) theory allows a geometric description in terms of extrinsic geometry of the worldsheet treated as a surface embedded into a flat $D$-dimensional Minkowski

\footnotetext{
* Lise Meitner Fellow of the "Fonds zur Förderung der wissenschaftlichen Forschung" at the Institut für Theoretische Physik, Technische Universität Wien, A-1040 Vienna Austria,

E-mail: bandos@tph32.tuwien.ac.at

${ }^{\dagger}$ E-mail: eivanov@thsun1.jinr.ru

${ }^{\ddagger}$ E-mail: alexandr@ff.dsu.dp.ua

$\S$ E-mail: theorph@ff.dsu.dp.ua
}

\section{Introduction}


space [1, 2, 3, 4⿴囗十。

In this approach the dynamics of a free relativistic string is described by the Maurer-Cartan equation supplemented with the additional conditions which insure the string worldsheet to be a minimal surface embedded into the flat target space-time. All these additional constraints can be solved algebraically, after which one is left with some $s o(1, D-1)$ valued connection form whose curvature vanishes due to the Maurer-Cartan equations [5]. Thus, though the string in the geometric approach is described by nonlinear equations [1, 2, 5], the latter are finally reduced to the zero curvature conditions for a $s o(1, D-1)$ valued connection form properly specified in terms of the independent field variables [5].

The system of independent equations describing a free string theory in the Minkowski space of arbitrary dimension $D$ was derived, for the first time, by Zheltukhin [1]. In the form when all the gauge symmetries inherent in the string theory are retained, this system is formed by the WZNW sigma-model-type equation

$$
\partial_{(--)}\left(\left(\partial_{(++)} G\right) G^{T}\right)^{i j}=e^{2 W} G^{[i \mid k} M_{(--)}^{(++) k} M_{(++)}^{(--) \mid j]}
$$

for the $S O(1, D-1)$ valued matrix field $G^{i j}$

$$
G G^{T}=I
$$

and the Liouville-type equation

$$
\partial_{(++)} \partial_{(--)} W=\frac{1}{4} M_{(++)}^{(--) i} G^{i j} M_{(--)}^{(++) j} e^{2 W}
$$

for a "scalar density" $e^{W}[5]$. Thus the considered system involves these two independent fields, as well as two chiral $S O(D-2)$ vector fields $M_{(++)}^{(--) i}, M_{(++)}^{(++) i}$,

$$
\partial_{(--)} M_{(++)}^{(--) j}=0, \quad \partial_{(++)} M_{(--)}^{(++) j}=0,
$$

which appear in the right hand side.

Already at the early stage of developing the geometric approach it was observed that at least for the low dimensions $D$ this system is exactly solvable. Indeed, it is reduced to the Liouville equation for $D=3$ and a complex Liouville equation for $D=4$. The general solution for $D=5$ was found in [2]. However, for the generic case the general solution of the equations (11), (2), (3) was unknown so far.

In this paper we present the general solution of these equations for any value of $D$.

First of all, our result gives a new example of non-trivial system of exactly solvable nonlinear equations.

Secondly, this opens a possibility to study the classical and quantum string theory in terms of new left- and right-moving variables which parametrize the general solution.

The meaning and origin of our results require some comments.

The standard equations of motion for the string theory become linear in the conformal gauge. The general solution of these linear equations is given by the sum of chiral functions

\footnotetext{
${ }^{1}$ See [3] for a supersymmetric generalization of the classical surface theory and its application to superstrings and $N=1$ superbranes.
} 
(subjected to the Virasoro constraints). The string-inspired nonlinear equations (i.e. the equations describing bosonic string theory in the geometric approach) encode the information about just the same dynamical system. Thus it is natural to expect (and, as we demonstrate here, this is indeed the case) that these equations are exactly solvable and that their general solution should be expressible in terms of chiral data.

Thus, the first step was to seek for an adequate set of chiral variables appropriate for constructing the general solution. It turned out that the necessary variables were provided by the harmonic approach [6] adapted to the case of Lorentz groups in refs. [7] - [11].

Namely, in ref. [8] it was found that the "constrained chiral twistor-like variables" (which can be identified with the spinor $S O(1, D-1)$ Lorentz harmonics [9, 10, 11]) can be used to obtain a covariant solution of the chiral Virasoro constraints

$$
\begin{gathered}
\partial_{(++)} X \frac{m}{L} \partial_{(++)} X \frac{m}{L}=0, \quad \partial_{(--)} X \frac{m}{R} \partial_{(--)} X \frac{m}{R}=0, \\
\partial_{(--)} X \frac{m}{L}=0, \quad \partial_{(++)} X \frac{m}{R}=0, \quad(\underline{m}=0,1 \ldots D-1)
\end{gathered}
$$

which are to be imposed on the solutions $X \frac{m}{L}, X \frac{m}{R}$ of the string equations of motion in the conformal gauge. The $D=3,4,6$ strings were treated in this way. It was analysed how the fields composed from such chiral twistors are related to the corresponding string-inspired nonlinear equations.

Here, instead of solving the chiral Virasoro constraints of $D$-dimensional string theory, we construct two chiral moving frames given by the two sets of chiral $S O(1, D-1)$ Lorentz harmonic variables [7], and identify the left- and right-moving vectors $\partial_{(++)} X_{L}^{\frac{m}{L}}$ and $\partial_{(--)} X_{R}^{\frac{m}{R}}$ with the light-like components of the relevant harmonic matrix. Each set of the moving frame variables parametrizes a special coset space of the group $S O(1, D-1)$. Despite the fact that the group $S O(1, D-1)$ on its own is non-compact, this coset space is compact and is isomorphic to the $D$-dimensional sphere [10]

$$
S^{D-2}=S O(1, D-1) /\left[S O(1,1) \times S O(D-2) \otimes K_{D-2}\right] .
$$

It turns out to provide us with the appropriate chiral data for constructing the general solution of the string-inspired nonlinear equations (11) - (3). We construct such a solution explicitly and argue that this is the general one.

\section{Basic notation}

Our conventions basically coincide with those of Refs. [5]. The indices $m, n, \ldots=0,1=$ $(++),(--)\left(v^{m} \equiv v^{ \pm \pm}=\left(v^{0} \pm v^{1}\right)\right)$ label the worldsheet vectors, while $\underline{m}, \underline{n}, \ldots=0,1, \ldots,(D-1)$ are the flat target space vector indices.

For the tangent space indices we use the notations $a, b, \ldots=0,1$ and $\underline{a}, \underline{b}, \ldots=0,1, \ldots,(D-1)$. Thus, in all cases, underlined indices correspond to the $D$-dimensional target space and the non-underlined ones refer to the two-dimensional $(d=2)$ worldsheet.

The indices,+- denote the weights of tangent space vectors and spinors with respect to both the worldsheet Lorentz group $S O(1,1)$ and the $S O(1,1)$ subgroup of the target space Lorentz group $S O(1, D-1)$. These two subgroups are identified with each other in the version of the "geometric approach" to string theory which forms the basis of the present consideration (see [3, 5] and refs. therein). E.g., we write $V^{a}=\left(V^{0}, V^{1}\right)=\left(\frac{1}{2}\left(V^{++}+V^{--}\right), \frac{1}{2}\left(V^{++}-V^{--}\right)\right)$ for the $d=2$ tangent space vectors (and reserve the notations $\psi^{\alpha}=\left(\psi^{+}, \psi^{-}\right.$) for the $d=2$ 
spinors which appear in supersymmetric generalizations of our approach). The indices,+within the parentheses denote the weights with respect to the $d=2$ conformal symmetry, e.g. $d \xi^{m}=\left(d \xi^{0}, d \xi^{1}\right)=\left(\frac{1}{2}\left(d \xi^{(++)}+d \xi^{(--)}\right), \frac{1}{2}\left(d \xi^{(++)}-d \xi^{(-)}\right)\right)$. The weights with respect to the chiral affine $S O(1,1)_{L}$ and $S O(1,1)_{R}$ transformations are indicated in the same way.

We use the subscripts $L$ and $R$ to denote the chiral functions of the string worldsheet coordinates $\xi^{m}=\left(\xi^{(++)}, \xi^{(--)}\right)$

$$
\begin{array}{cc}
f_{L}=f_{L}\left(\xi^{(++)}\right), & f_{R}=f_{R}\left(\xi^{(--)}\right), \\
\partial_{(--)} f_{L}=0, & \partial_{(++)} f_{R}=0 .
\end{array}
$$

They should not be confused with the calligraphic subscripts $\mathcal{L}$ and $\mathcal{R}$ carried by some fields. The chiral Lorentz harmonics (chiral moving frame variables) are denoted by the letters $l$ and $r$, while the generic (non-chiral) Lorentz harmonics are denoted by $u$.

\section{Geometric approach to string dynamics and string-inspired nonlinear equations}

\subsection{Lorentz harmonics}

We begin with the definition of the moving frame variables (Lorentz harmonics [6, 7]) which are the basic ingredients of the geometric approach to $D$-dimensional bosonic string theory

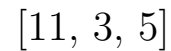

$$
\begin{gathered}
u_{\underline{\underline{m}}}^{\underline{a}}(\xi) \equiv\left(\frac{1}{2}\left(u_{\underline{m}}^{++}+u_{\underline{m}}^{--}\right), \quad u_{\underline{m}}^{i}, \frac{1}{2}\left(u_{\underline{m}}^{++}-u_{\underline{m}}^{--}\right)\right) \\
\underline{m}=0,1, \ldots, D-1, \quad \underline{a}=0,1, \ldots, D-1 .
\end{gathered}
$$

These objects are subject to the following orthonormality conditions

$$
\begin{aligned}
& u \underline{\underline{m}} u_{\underline{m b}}=\eta_{\underline{a b}} \equiv \operatorname{diag}(1,-1, \ldots,-1), \\
& \Leftrightarrow\left\{\begin{array}{l}
u_{m}^{++} u^{\underline{\underline{m}}++}=0, \quad u_{\underline{m}}^{--} u^{\underline{\underline{m}}--}=0, \\
u_{\underline{m}}^{++} u^{\underline{\underline{m}}--}=2, \\
u_{\underline{m}}^{++} u^{\underline{\underline{m}} i}=0, \quad u_{\underline{m}}^{--} u^{\underline{\underline{m}} i}=0, \\
u_{\underline{m}}^{i} u^{\underline{\underline{m}} j}=-\delta^{i j} .
\end{array}\right.
\end{aligned}
$$

They imply that the $D \times D$ matrix $u_{\underline{\underline{m}}} \underline{\underline{a}}$ (4) belongs to the group $S O(1, D-1)$

$$
u_{\underline{m}}^{\underline{a}}(\xi) \quad \in \quad S O(1, D-1) .
$$

The completeness condition

$$
u_{\underline{\underline{m}}}^{\underline{a}} u_{\underline{n a}} \equiv \frac{1}{2} u_{\underline{m}}^{++} u_{\underline{n}}^{--}+\frac{1}{2} u_{\underline{m}}^{--} u_{\underline{n}}^{++}-u_{\underline{m}}^{i} u_{\underline{n}}^{i}=\eta_{\underline{m n}} \equiv \operatorname{diag}(1,-1, \ldots,-1)
$$

follows from Eq. (5). In Eqs. (14), (7) the light-like notation is used

$$
\begin{aligned}
& u_{\underline{m}}^{++} \equiv u_{\underline{m}}^{0}+u_{\underline{m}}^{D-1}=u_{0 \underline{m}}-u_{D-1 \underline{m}}=u_{--\underline{m}}, \\
& u_{\underline{m}}^{--} \equiv u_{\underline{m}}^{0}-u_{\underline{m}}^{D-1}=u_{0 \underline{m}}+u_{D-\underline{m}}=u_{++\underline{m}} .
\end{aligned}
$$


The constraints (5), (77) as they stand are invariant under the local $S O(1, D-1)$ transformations acting on the tangent space indices $\underline{a}, \underline{b}, \ldots$. Below we will see that for the purpose of constructing the geometric approach description of the string theory this symmetry should be restricted to $S O(1,1) \otimes S O(D-2)$ in accordance with the splitting (雨). Just this local symmetry is respected by the basic geometric postulate of such a description, namely by the condition that the Lorentz harmonic frame be adapted to the string worldsheet (see Eq. (23) below). This means that two of $(D-2)$ vectors $u_{\underline{\underline{a}}}$ are chosen to be tangent to the worldsheet while the remaining ones $u_{\underline{m}}^{i}$ are orthogonal to it. The local (gauge) symmetry $S O(1,1) \otimes S O(D-2)$ reflects, respectively, the freedom of the $d=2$ Lorentz rotation of the vectors $u_{\underline{m}}^{0,(D-1)}$ tangent to the worldsheet and $S O(D)$ rotations of the vectors $u_{\underline{m}}^{i}$ orthogonal to the worldsheet (in the light-like notation $(8)$ the $d=2$ rotations are reduced to the opposite weight scaling transformations of the vectors $u^{++}$and $u^{--}$).

Thus the vectors $u \frac{a}{\underline{m}}$ appropriate for the description of the external geometry of the bosonic string worldsheet parametrize the non-compact coset space

$$
\frac{S O(1, D-1)}{S O(1,1) \times S O(D-2)} .
$$

In other words, the harmonics (4) regarded as the worldsheet fields define a map of the worldsheet $\mathcal{M}^{(1,1)}=\left\{\xi^{m}\right\}$ onto the non-compact coset (9)

$$
u \underline{\underline{m}} \quad: \quad \mathcal{M}^{(1,1)}=\left\{\xi^{m}\right\} \rightarrow \frac{S O(1, D-1)}{S O(1,1) \times S O(D-2)} .
$$

Below we will see that the basic ingredients of the sought general solution of the stringinspired equations will be smaller sets of left- and right-moving Lorentz harmonics parametrizing some compact subspaces in the chiral copies of the coset space (9).

\subsubsection{Cartan 1-forms}

Differentials of the harmonic variables can be calculated with taking into account the conditions (4). Differentiating Eq. (四) produces the equation

$$
d u \underline{\underline{a}} u \frac{b m}{\underline{m}}+u \frac{a m}{\underline{a}} d u \underline{\underline{b}}=0
$$

which can be solved as follows

$$
d u_{\underline{\underline{m}}}^{\underline{a}}=u_{\underline{\underline{m}}}^{\underline{\underline{b}}} \Omega_{\underline{b}} \underline{\underline{a}}(d) \quad \Leftrightarrow \quad\left\{\begin{array}{l}
d u_{\underline{m}}^{++}=u_{\underline{m}}^{++} \omega+u_{\underline{m}}^{i} f^{++i}(d), \\
d u_{\underline{m}}^{-}-u_{\underline{m}}^{--} \omega+u_{\underline{m}}^{i} f^{--i}(d), \\
d u_{\underline{m}}^{i}=-u_{\underline{m}}^{j} A^{j i}+\frac{1}{2} u_{\underline{m}}^{++} f^{--i}(d)+\frac{1}{2} u_{\underline{m}}^{--} f^{++i}(d) .
\end{array}\right.
$$

Here

$$
\Omega_{\underline{b}}^{\underline{a}} \equiv u_{\underline{\underline{b}}}^{\underline{m}} d u \underline{\underline{m}}=\left(\begin{array}{ccc}
\omega & 0 & \frac{1}{\sqrt{2}} f^{--i}(d) \\
0 & -\omega & \frac{1}{\sqrt{2}} f^{++i}(d) \\
\frac{1}{\sqrt{2}} f^{++i}(d) & \frac{1}{\sqrt{2}} f^{--i}(d) & A^{j i}(d)
\end{array}\right), \quad \Omega^{\underline{a b}} \equiv \eta \underline{a c} \Omega_{\underline{\underline{b}} \underline{b}}=-\Omega^{\underline{b a}}
$$

are the $S O(1, D-1)$ Cartan forms (in the vector representation of the $S O(1, D-1)$ generators). Due to $(\mathbb{\Psi})$, they are naturally divided into the $S O(1,1) \times S O(D-2)$ covariant forms

$$
f^{++i} \equiv u_{\underline{m}}^{++} d u^{\underline{m}} i
$$




$$
f^{--i} \equiv u_{\underline{m}}^{--} d u^{\underline{m}} i
$$

which constitute a vielbein of the non-compact coset space $\frac{S O(1, D-1)}{S O(1,1) \otimes S O(D-2)}$, the $S O(1,1)$ (spin) connection

$$
\omega \equiv \frac{1}{2} u_{\underline{m}}^{--} d u^{\underline{m}}++
$$

and the $S O(D-2)$ connections (gauge fields)

$$
A^{i j} \equiv u_{\underline{m}}^{i} d u^{\underline{m}} j \equiv-u_{\underline{m}}^{j} d u^{\underline{m} i} .
$$

\subsubsection{Parabolic subgroup}

As was mentioned above, the choice of the tangent space local group as $S O(1,1) \otimes S O(D)$ (and the coset space (9) for Lorentz harmonics) is motivated by the adaptation postulate which "solders" harmonics to the worldsheet and is so relevant just to the geometric description of strings. Formally, the same harmonic (moving frame) variables (4) can be used to give a geometric description of the massless particle. But in this case the adaptation of the moving frame would consist in requiring that one of the light-like vectors, e.g. $u_{m}^{++}$, is tangent to the worldline, while $u^{i}$ and $u^{--}$are orthogonal to it. Such an adaptation is covariant with respect to the following right gauge transformations [10] (see also [9], where the Hamiltonian form of the corresponding $D=4$ transformations has been presented)

$$
\begin{aligned}
u_{\underline{m}}^{++\prime} & =u_{\underline{m}}^{++} V^{-1}, \\
u_{\underline{m}}^{i \prime} & =\left(u_{\underline{m}}^{++} V^{--j}+u_{\underline{m}}^{j}\right) V^{j i}, \\
u_{\underline{m}}^{--\prime} & =\left(u_{\underline{m}}^{--}+u_{\underline{m}}^{++} V^{--i} V^{--i}+2 u_{\underline{m}}^{i} V^{--j}\right) V .
\end{aligned}
$$

The transformations (17) form the maximal proper subgroup (parabolic subgroup) $S O(1,1) \times$ $S O(D-2) \propto K_{D-2}$ of the Lorentz group $S O(1, D-1)$ [10. An arbitrary element of this subgroup is characterized by the $S O(1,1)$ transformation $V=e^{\alpha}$, the matrix $V^{i j}$ of $S O(D-$ 2 )-orthogonal rotations and the parameters $V^{--i}$ of the boosts $K_{D-2}$. Thus, in the case of geometric description of massless particle, as well as in any case when the adaptation of the

moving frame involves only one light-like moving frame vector, the harmonics $u \underline{\underline{m}}$ (4) can be regarded as parameters of the compact coset space

$$
S^{D-2}=\frac{S O(1, D-1)}{S O(1,1) \times S O(D-2) \otimes K_{D-2}} .
$$

It is isomorphic to a $(D-2)$-dimensional sphere $S^{D-2}$ [10.

The Cartan forms (13), (14), (15), and (16) are transformed under (17) as follows

$$
\begin{gathered}
f^{++i \prime}=f^{++j} V^{j i} V^{-1} \\
f^{--i \prime}=\left(f^{--j}+2 \mathcal{D} V^{--j}-2 f^{++k}\left(V^{--k} V^{--j}-\frac{1}{2} \delta^{k j} V^{--l} V^{--l}\right)\right) V^{j i}, \\
\mathcal{D} V^{--j} \equiv d V^{--j}+\omega V^{--j}-V^{--k} A^{k j} \\
\omega^{\prime}=\omega-f^{++i} V^{--i}+V d V^{-1},
\end{gathered}
$$




$$
A^{i j \prime}=\left(V^{-1} A V\right)^{i j}-\left(V^{-1} d V\right)^{i j}-2 f^{++k} V^{k[i \mid} V^{--l} V^{l \mid j]} .
$$

Though the $K_{D-2}$ transformations (17) with the parameters $V^{--i}$ are not the gauge symmetries of the whole bosonic string theory, they play a crucial role for understanding the group-theoretical structure of the general solution of the nonlinear equations (1), (8) (see Section 3) 2. Moreover, to define the general solution, we introduce two chiral sets of moving frame variables, $l_{\underline{m}}^{(\underline{a})}$ and $r_{\underline{\underline{a}}}^{(\underline{a})}$ (see Section 2.2). Their "adaption" is realized just in the "particle-like" fashion and, thus, respects covariance under the chiral counterparts of the maximal parabolic symmetry (17) (cf. (82), (83)).

\subsection{The first-order form of string equations and the geometric approach to string theory}

The Lorentz harmonics give us a possibility to rewrite the string equations of motion in the first order form, namely, as the following set of equations [11, 3, 5]

$$
\begin{gathered}
d X^{\underline{m}}=\frac{1}{2} e^{++} u^{--\underline{m}}+\frac{1}{2} e^{--} u^{++} \underline{m}, \\
d\left(e^{++} u^{--\underline{m}}-e^{--} u^{++\underline{m}}\right)=0 .
\end{gathered}
$$

Here $e^{ \pm \pm}=d \xi^{m} e_{m}^{ \pm \pm}$is a worldsheet vielbein. While dealing with (23) and (24), one should take into account the restrictions (11) on the differentials of the harmonic variables.

Eq. (23) is the adaptation relation already referred to in the consideration above. It plays the basic role in the geometric approach to strings. In particular, it implies that the intrinsic worldsheet metric is identified with the induced one

$$
\frac{1}{2}\left(e_{m}^{++} e_{n}^{--}+e_{m}^{--} e_{n}^{++}\right)=g_{m n}=\partial_{m} X^{\underline{m}} \partial_{n} X_{\underline{m}} .
$$

The geometric meaning of Eq. (23) consists in the statement that the string worldsheet is a surface embedded into the $D$-dimensional Minkowski space-time. On its own right, it has no any dynamical content and gives rise to purely geometric consequences.

The integrability conditions $(d d X \equiv 0)$ for Eq. (23) are as follows

$$
\begin{gathered}
T^{++} \equiv d e^{++}+e^{++} \wedge \omega=0, \quad T^{--} \equiv d e^{--}-e^{--} \wedge \omega=0, \\
e^{++} \wedge f^{--i}+e^{--} \wedge f^{++i}=0 \quad \Leftrightarrow \quad f_{--}^{--i}-f_{++}^{++i}=0 .
\end{gathered}
$$

Thus they require the torsion $2-$ forms $T^{ \pm \pm}$to vanish, thereby imposing proper constraints on the induced spin connection $\omega$. Besides, they imply the $S O(1,1)$ invariant components of the covariant one-forms $f^{++i}$ and $f^{--i}$ to coincide with each other

$$
f_{--}^{--i}=f_{++}^{++i}=\frac{1}{2} h^{i}
$$

The quantity $h^{i}$ can be easily recognized as the main curvatures of the embedded surface [4]. Indeed, using Eq. (23) to express the light-like harmonic vectors in terms of derivatives of the embedding functions $X$, we arrive at the standard expression for the main curvatures

$$
h^{i}=g^{m n} K_{m n}^{i}, \quad K_{m n}^{i}=-\partial_{m} \partial_{n} X^{\underline{m}} u_{\underline{m}}^{i} .
$$

\footnotetext{
${ }^{2}$ It was noted in [5] that the boost symmetry allows one to introduce a non-trivial dependence on a spectral parameter into the connection 1-forms entering the zero curvature representation for the nonlinear equations (11), (2).
} 


\subsubsection{Minimal embedding}

Using Eq. (11), one finds that Eq. (24) implies the vanishing of the main curvatures (28)

$$
h^{i}=0
$$

Thus the surface defined by Eq. (24) is minimal [4].

On the other hand, expressing all the auxiliary variables $u$ and $e$ through the derivatives of the embedding functions $X(\xi)$ and using the induced metric (25)

$$
g_{m n} \equiv \partial_{m} X \underline{m} \partial_{n} X_{\underline{m}},
$$

and its inverse $g^{m n}$, one can rewrite Eq. (30) (or (24)) in the form

$$
\partial_{m}\left(\sqrt{-g} g^{m n} \partial_{n} X^{\underline{m}}\right)=0
$$

which is the standard string equations of motion pertinent to the Nambu-Goto action (see [5] for details). Thus the strings dynamics in the geometric approach is contained just in eq. (24).

Note that it is important for the geometric approach description that the minimal embedding is described by the covariant Cartan forms $f^{++i}, f^{--i}$ containing in their decomposition only one of the two basic forms $e^{--}, e^{++}$

$$
f^{++i}=e^{--} f_{--}^{++i}, \quad f^{--i}=e^{++} f_{++}^{-i}
$$

(cf. Eqs. (28), (30)). Actually, Eqs. (33) encode three previous equations: (27), (28) and (30). Thus the string dynamics is finally encoded just in Eqs. (33).

\subsection{Maurer-Cartan equation and the string-inspired nonlinear equations}

The integrability conditions for Eqs. (11) produce the Maurer-Cartan equations

$$
d \Omega \underline{\underline{a b}}-\Omega^{\underline{a}} \underline{\underline{c}} \wedge \Omega \underline{c b}=0 .
$$

Owing to Eq. (4), Eqs. (34) naturally split into the following equations for the coset vielbeins $f^{ \pm \pm i}(13),(14)$ and the connection one-forms $\omega, A^{i j}$ (15), (16)

$$
\begin{gathered}
\mathcal{D} f^{++i} \equiv d f^{++i}-f^{++i} \wedge \omega+f^{++j} \wedge A^{j i}=0 \\
\mathcal{D} f^{--i} \equiv d f^{--i}+f^{--i} \wedge \omega+f^{--j} \wedge A^{j i}=0 \\
\mathcal{R} \equiv d \omega=\frac{1}{2} f^{--i} \wedge f^{++i}, \\
R^{i j} \equiv d A^{i j}+A^{i k} \wedge A^{k j}=-f^{--[i} \wedge f^{++j]}
\end{gathered}
$$

Eqs. (35) - (38) amount to the Peterson-Codazzi, Gauss and Ricci equations of the classical surface theory [四].

Thus, in the geometric approach framework, the dynamics of string is described by the vielbein $e^{ \pm \pm}$and the set of Cartan forms $\omega, f^{ \pm \pm i}, A^{i j}$ which satisfy Eqs. (26), (33) and the Maurer-Cartan Eqs. (35) - 38) [4, 1, 2, 3, 5. 
The most essential general feature of this approach is that Eqs. (33), (26), (35), (36) can be solved algebraically [5].

Indeed, using the fact that any connection is integrable on any one-dimensional subspace, we can specify the expressions for the $S O(1,1)$ and $S O(D-2)$ connection one-forms in the following way

$$
\begin{gathered}
\omega=e^{++} \nabla_{++}(W-L)-e^{--} \nabla_{--}(W+L), \\
A^{i j}=e^{++} \nabla_{++} G_{\mathcal{R}}^{i k} G_{\mathcal{R}}^{j k}+e^{--} \nabla_{--} G_{\mathcal{L}}^{i k} G_{\mathcal{L}}^{j k},
\end{gathered}
$$

where $G_{\mathcal{L}}$ and $G_{\mathcal{R}}$ are some $S O(D-2)$ group matrices,

$$
G_{\mathcal{L}} G_{\mathcal{L}}^{T}=I, \quad G_{\mathcal{R}} G_{\mathcal{R}}^{T}=I .
$$

Eqs. (39), (40) provide a possibility to rewrite Eqs. (26), (35), (36) as the conditions of closeness of some one-forms

$$
\begin{gathered}
d\left(e^{++} \exp (W+L)\right)=0, \quad d\left(e^{--} \exp (W-L)\right)=0 . \\
d\left(f^{++i} G_{\mathcal{R}}^{j i} \exp (-W+L)\right)=0, \quad d\left(f^{--i} G_{\mathcal{L}}^{j i} \exp (-W-L)\right)=0
\end{gathered}
$$

(Eqs. (33) have been used when deriving (43)).

The general solution of Eqs. (42) (up to some possible topological subtleties which are unessential for the present study) is provided by

$$
e^{++}=d \xi^{(++)} M_{(++)}^{(++)}\left(\xi^{(++)}\right) \exp (-W-L), \quad e^{--}=d \xi^{(--)} M_{(--)}^{(--)}\left(\xi^{(--)}\right) \exp (-W+L)
$$

Here $\xi^{( \pm \pm)}$are some functions of the string worldsheet coordinates with the only defining demand that their differentials are linearly-independent one-forms. It is convenient, however, to choose $\xi^{( \pm \pm)}$as a set of local coordinates on the worldsheet

$$
\xi^{m}=\xi^{( \pm \pm)}
$$

This choice fixes the gauge with respect to the worldsheet reparametrizations (general coordinate transformations) so that only 2-dimensional conformal reparametrizations survive (see Eq. (61) below).

In the holonomic basis $d \xi^{( \pm \pm)}$the components of the vielbein form $e^{ \pm \pm}$are given by

$$
\begin{array}{ll}
e_{(++)}^{++}=M_{(++)}^{(++)} \exp (-W-L), & e_{(--)}^{++}=0, \\
e_{(++)}^{--}=0, & e_{(--)}^{--}=M_{(--)}^{(--)} \exp (-W+L), \\
e_{--}^{(++)}=0, & e_{--}^{(--)}=2\left(M_{(--)}^{(--)}\right)^{-1} \exp (W-L), \\
e_{++}^{(++)}=2\left(M_{(++)}^{(++)}\right)^{-1} \exp (W+L), & e_{++}^{(--)}=0,
\end{array}
$$

the induced metric (25) is conformally flat

$$
d s^{2}=d \xi^{m} d \xi^{n} g_{m n}=d \xi^{(++)} d \xi^{(--)} M_{(++)}^{(++)} M_{(--)}^{(--)} e^{2 W}
$$

and the covariant derivatives are proportional to the corresponding holonomic ones

$$
e^{++} \nabla_{++}=d \xi^{(++)} \partial_{(++)}, \quad e^{--} \nabla_{--}=d \xi^{(--)} \partial_{(--)}
$$


Hence, the expressions (39) and (40) for the gauge connections can be rewritten as follows

$$
\begin{gathered}
\omega=d \xi^{(++)} \partial_{(++)}(W-L)-d \xi^{(--)} \partial_{(--)}(W+L) \\
A^{i j}=d \xi^{(++)} \partial_{(++)} G_{\mathcal{R}}^{i k} G_{\mathcal{R}}^{j k}+d \xi^{(--)} \partial_{(--)} G_{\mathcal{L}}^{i k} G_{\mathcal{L}}^{j k}
\end{gathered}
$$

Finally, using the holonomic basis, one can write down the general solution of the PetersonCodazzi equations (43) for the covariant forms (33) as

$$
\begin{aligned}
& f^{++i}=d \xi^{(--)} e^{W-L} G_{\mathcal{R}}^{i j} M_{(--)}^{(++) j}\left(\xi^{(--)}\right), \\
& f^{--i}=d \xi^{(++)} e^{W+L} G_{\mathcal{L}}^{i j} M_{(++)}^{(--) j}\left(\xi^{(++)}\right),
\end{aligned}
$$

where vector fields $M_{(--)}^{(++)}, M_{(++)}^{(--) j}$ are chiral, similarly to the parameters $M_{(++)}^{(++)}, M_{(--)}^{(--)}$of the solutions (44) of Eqs. (26)

$$
\partial_{(++)} M_{(--)}^{(++) j}=0, \quad \partial_{(--)} M_{(++)}^{(--) j}=0 .
$$

Thus, following [5], we have solved algebraically all the equations except for the Gauss and Ricci ones (37), (38). Substituting (49), (50), (51), (52) into Eqs. (37) and (38), we obtain the set of nonlinear equations

$$
\begin{gathered}
\partial_{(++)} \partial_{(--)} W=\frac{1}{4} M_{(--)}^{(++) i}\left(G_{\mathcal{L}}^{T} G_{\mathcal{R}}\right)^{i j} M_{(++)}^{(--) j} e^{2 W} \\
\partial_{(--)}\left(\left(\partial_{(++)} G_{\mathcal{L}}\right) G_{\mathcal{L}}^{T}\right)^{i j}-\partial_{(++)}\left(\left(\partial_{(--)} G_{\mathcal{R}}\right) G_{\mathcal{R}}^{T}\right)^{i j}+\left[\left(\partial_{(++)} G_{\mathcal{L}}\right) G_{\mathcal{L}}^{T},\left(\partial_{(--)} G_{\mathcal{R}}\right) G_{\mathcal{R}}^{T}\right]^{i j}= \\
=e^{2 W}\left(G_{\mathcal{L}} M_{(--)}^{(++)}\right)^{[i}\left(G_{\mathcal{R}} M_{(++)}^{(--)}\right)^{j]}
\end{gathered}
$$

describing the extrinsic geometry of the string worldsheet embedded into a D-dimensional Minkowski space.

\subsection{Zero curvature representation and the associated linear system}

As we saw, most of the equations of the geometric approach (33), (26), (35), (36) can be solved algebraically and the final set of the string-inspired nonlinear equations (53), (54) emerges as the result of substitution of these algebraic solutions into the Gauss and Ricci equations (37), (38). Since the latter constitute a part of the Maurer-Cartan equation (34), one can conclude that the zero curvature representation for the nonlinear equations (53), (54) is given by the Maurer-Cartan equation (34) for the $s o(1, D-1)$ valued connection one-forms specified by Eqs. (12), (49), (50), (51), (52).

The associated linear system is provided by Eq. (11) with the forms (34) specified by Eqs. (12), (49), (50), (51), (52).

A non-trivial dependence on a spectral parameter can be introduced into the associated linear system by means of the parabolic subgroup transformations (17) [5].

Thus the nonlinear equations (53), (54) possess all the features inherent to the equations which can be solved by the Inverse Scattering Method [13. Below we will prove that they are solvable even in a more strong sense, like the Liouville, Toda or WZNW sigma-model equations. Namely, we will deduce an explicit form of the general solution. It is interesting that the solution can be obtained by exploiting the parabolic group transformations (17). We will demonstrate this in the last section of the present paper. 


\subsection{Symmetries and bridges}

The obtained nonlinear equations and their zero curvature representation (34), (12), (49), (50), (51), (52) possess a number of powerful symmetries.

As was already mentioned, the local (gauge) symmetries of the string model form the $S O(1,1) \times S O(D-2)$ group

$$
\begin{gathered}
\omega^{\prime}=\omega+V d V^{-1}=\omega+\alpha, \\
A^{i j \prime}=\left(V^{-1} A V\right)^{i j}-\left(V^{-1} d V\right)^{i j}, \\
f^{++i \prime}=V^{-1} f^{++j} V^{j i}, \\
f^{--i \prime}=V f^{--j} V^{j i} .
\end{gathered}
$$

The matrix fields $G_{\mathcal{L}, \mathcal{R}}$ appearing in Eqs. (51), (52) and (50) are transformed homogeneously under the $S O(D-2)$ gauge symmetry

$$
G_{\mathcal{L}, \mathcal{R}}^{i j \prime}=G_{\mathcal{L}, \mathcal{R}}^{k j} V^{k i}=\left(V^{-1}\right)^{i k} G_{\mathcal{L}, \mathcal{R}}^{k j},
$$

whereas the field $L$ is pure gauge

$$
L^{\prime}=L-\alpha, \quad V=e^{-\alpha} .
$$

In other words, it is a compensator (or Nambu-Goldstone field) for the $S O(1,1)$ gauge symmetry.

Examining the expressions (44), (51), and (52), one concludes that our system possesses two types of the infinite-dimensional global symmetries whose parameters can be combined into left-moving and right-moving (chiral) functions. One of them is the $d=2$ conformal symmetry

$$
\begin{gathered}
d \xi^{(++) \prime}=d \xi^{(++)} s_{L}\left(\xi^{(++)}\right), \quad d \xi^{(--) \prime}=d \xi^{(--)} s_{R}\left(\xi^{(--)}\right), \\
\partial_{(--)} s_{L}=0, \quad \partial_{(++)} s_{R}=0 .
\end{gathered}
$$

The second one is realized as chiral rescalings of the chiral fields $M_{(++)}^{(++)}$and $M_{(--)}^{(--)}$present in Eqs. (51), (52)

$$
\begin{array}{cl}
M_{(++)}^{(++) \prime}=M_{(++)}^{(+)} e^{h_{L}}, & M_{(--)}^{(--) \prime}=M_{(--)}^{(-)} e^{h_{R}}, \\
\partial_{(--)} h_{L}=0, & \partial_{(++)} h_{R}=0 .
\end{array}
$$

Clearly, these rescalings can be treated as a sort of affine (or Kac-Moody) $S O(1,1)_{L}$ and $S O(1,1)_{R}$ symmetry transformations (i.e. as $S O(1,1)$ transformations with the parameters depending on $\xi^{(++)}$and $\xi^{(--)}$, respectively). by

The full set of non-trivial transformations of these symmetries on the involved fields is given

$$
\begin{aligned}
M_{(++)}^{(++) \prime}=M_{(+)}^{(++)} s_{L}^{-1} e^{h_{L}}, & M_{(--)}^{(--) \prime}=M_{(--)}^{(-)} s_{R}^{-1} e^{h_{R}}, \\
(W+L)^{\prime}=(W+L)+h_{L}-\alpha, & (W-L)^{\prime}=(W-L)+h_{R}+\alpha, \\
\left(M_{(+)}^{(--) i}\right)^{\prime}=M_{(++)}^{(--) i} s_{L}^{-1} e^{-h_{L}}, & \left(M_{(--)}^{(++) i}\right)^{\prime}=M_{(--)}^{(+) i} s_{R}^{-1} e^{-h_{R}} .
\end{aligned}
$$

We observe that the chiral fields $M_{(++)}^{(++)}, M_{(--)}^{(--)}$can be regarded as the "bridges" relating the affine $S O(1,1)_{L}, S O(1,1)_{R}$ groups to the corresponding chiral parts of the 2-dimensional conformal group, while

$$
e^{W+L}=\left(e^{W+L}\right)_{++}^{(++)}, \quad e^{W-L}=\left(e^{W-L}\right)_{--}^{(--)}
$$


as the bridges relating affine $S O(1,1)_{L}$ and $S O(1,1)_{R}$ to the gauge $S O(1,1)$ symmetry. Since the symmetries (61), (62) offer the possibility to choose the gauge $3 M_{(++)}^{(++)}=M_{(--)}^{(--)}=1$, in what follows, for simplicity, we will make no distinction between the $S O(1,1)_{L, R}$ indices and conformal ones. We will also use the superscript (--) instead of the $S O(1,1)_{L}$ subscript $(++)$ for the chiral vector field $M_{(++)}^{(--) i}$ (the chirality of the latter field, $\partial_{(--)} M_{(++)}^{(--) i}=0$, excludes any confusion).

In addition, our equations possess an invariance under right multiplication of the $S O(D-2)$ valued fields $G_{\mathcal{L}}$ and $G_{\mathcal{R}}$ by chiral $S O(D-2)$ matrices $H_{R}$ and $H_{L}$. So, the complete form of the appropriate symmetry transformations is

$$
\begin{aligned}
& G_{\mathcal{L}}^{\prime}=V^{-1} G_{\mathcal{L}} H_{L}, \quad G_{\mathcal{R}}^{\prime}=V^{-1} G_{\mathcal{R}} H_{R}, \\
& M_{(++)}^{(--) i \prime}=H_{L}^{i j} M_{(--)}^{(++) j}, \quad M_{(++)}^{(--) j \prime}=H_{R}^{i j} M_{(++)}^{(--) j}, \\
& H_{L} H_{L}^{T}=H_{R} H_{R}^{T}=I, \quad \partial_{(--)} H_{L}=\partial_{(++)} H_{R}=0 .
\end{aligned}
$$

Thus the orthogonal matrix fields $G_{\mathcal{L}}$ and $G_{\mathcal{R}}$ can be regarded as bridges between the gauge $S O(D-2)$ transformations and affine chiral $S O(D-2)_{L}$ and $S O(D-2)_{R}$ transformations, respectively.

\subsection{Simplified form of the nonlinear equations}

The system of nonlinear equations (53), (54) can be significantly simplified by using the $S O(D-$ 2) gauge symmetry with parameters $V^{i j}$ to fix a gauge

$$
G_{\mathcal{L}}=1, \quad G_{\mathcal{R}}=G .
$$

Then the sigma-model-type equation (54) acquires the simplest WZNW sigma-model-type form (1)

$$
\partial_{(--)}\left(\left(\partial_{(++)} G\right) G^{T}\right)^{i j}=e^{2 W} G^{[i \mid k} M_{(--)}^{(++) k} M_{(++)}^{(--) \mid j]}
$$

whereas the Liouville-type equation (53) becomes (1)

$$
\partial_{(++)} \partial_{(--)} W=\frac{1}{4} M_{(++)}^{(--) i} G^{i j} M_{(--)}^{(++) j} e^{2 W} .
$$

The gauge (67) is invariant under the action of two chiral affine $S O(D-2)$ symmetries with the parameters $H_{L}\left(\xi^{(++)}\right)$and $H_{R}\left(\xi^{(--)}\right)$, action of which on the matrix field $G$ is given by

$$
G^{\prime}=H_{L}^{-1} G H_{R}, \quad \partial_{(--)} H_{L}=\partial_{(++)} H_{R}=0 .
$$

\section{General solution of the string-inspired nonlinear equations}

\subsection{Standard string equations of motion, their solution and Virasoro constraints}

We start by discussing the familiar string equations of motion and Virasoro conditions in the standard setting. The study of the relationship of the solutions of these equation with the

\footnotetext{
${ }^{3}$ They also make it possible to fix the value of the norm of the chiral vector fields $M_{(\mp \mp)}^{ \pm \pm i}$ (see [5]).
} 
Lorentz harmonics (which, as was mentioned above, provide the associated linear system for the considered nonlinear equations) opens a possibility to construct the general solution of the nonlinear equations (69), (68).

The equations of motion of $D$-dimensional bosonic string following from the Nambu-Goto action (see [12] and Refs. therein) has the form (32)

$$
\partial_{m}\left(\sqrt{-g} g^{m n} \partial_{n} X^{\underline{m}}\right)=0
$$

where (recall eq. (31))

$$
g_{m n} \equiv \partial_{m} X^{\underline{m}} \partial_{n} X_{\underline{m}}
$$

is the induced metric, $g^{m n}$ is its inverse and $g=\operatorname{det}\left(g_{m n}\right)$.

In the conformal gauge (see, e.g. [12]) the string equation (71) becomes linear

$$
\tilde{\partial}_{(++)} \tilde{\partial}_{(--)} X^{\underline{m}} \equiv \frac{\partial}{\partial \tilde{\xi}^{(++)}} \frac{\partial}{\partial \tilde{\xi}^{(--)}} X^{\underline{m}}=0
$$

and has a general solution

$$
X^{\underline{m}}=X_{\frac{m}{L}}+X_{\frac{m}{R}}, \quad \tilde{\partial}_{(--)} X_{L}^{\frac{m}{L}}=0, \quad \tilde{\partial}_{(++)} X^{\frac{m}{R}}=0 .
$$

The chiral functions $X_{\frac{m}{L}}\left(\xi^{(\tilde{+}+)}\right), X_{R}^{\frac{m}{R}}\left(\xi^{(\tilde{-}-)}\right)$ are subjected to the Virasoro constraints

$$
\tilde{\partial}_{(++)} X \frac{m}{L} \tilde{\partial}_{(++)} X_{L \underline{m}}=0, \quad \tilde{\partial}_{(--)} X \frac{m}{R} \tilde{\partial}_{(--)} X_{R \underline{m}}=0 \text {. }
$$

Let us compare the solution (74) with the expressions (23) and (44) obtained in the geometric approach

$$
d X^{\underline{m}}=\frac{1}{2} d \xi^{(++)} e^{-W-L} M_{(++)}^{(++)} u^{--\underline{m}}+\frac{1}{2} d \xi^{(--)} e^{-W+L} M_{(--)}^{(--)} u^{++\underline{m}} .
$$

Since, due to Eq. (44), the induced metric is conformally flat in the coordinate frame $\xi^{( \pm \pm)}$, we can identify these worldsheet coordinates with the "conformal coordinates" $\tilde{\xi}^{( \pm \pm)}$used in the standard string description $(71)-(75)$

$$
\xi^{( \pm \pm)}=\tilde{\xi}^{( \pm \pm)}
$$

Thus Eqs. (76) and (74) result in

$$
\begin{aligned}
& \partial_{(++)} X^{\frac{m}{L}\left(\xi^{(++)}\right)}=\frac{1}{2} e^{-W-L} M_{(++)}^{(++)}\left(\xi^{(++)}\right) u^{--\underline{m}}, \\
& \partial_{(--)} X^{\frac{m}{R}}\left(\xi^{(--)}\right)=\frac{1}{2} e^{-W+L} M_{(--)}^{(--)}\left(\xi^{(--)}\right) u^{++\underline{m}} .
\end{aligned}
$$

It is easy to verify that the Virasoro constraints (75) are satisfied for the functions (77).

It is worth noticing that the l.h.s. of Eqs. (77) includes the chiral functions only (cf. (74)), while the r.h.s. involve the functions $W, L, u^{ \pm \pm}$which from the very beginning were assumed to depend on both coordinates $\xi^{( \pm \pm)}$.

We will demonstrate below that the origin of this fact lies in that any solution of the string equation produces a solution of the string-inspired nonlinear equations, i.e. the equations (53), (68) which describe the extrinsic geometry of the string worldsheet. 


\subsection{Chiral harmonics}

In ref. [8] it was discussed how to find an appropriate set of chiral functions for obtaining an explicit expression for the $W$ and $G^{i j}=\left(G^{-1}\right)^{j i}$ fields which enter the nonlinear equations (53), (68). Constrained twistors have been proposed as such variables for the case of bosonic string theories in dimensions $D=3,4,6$. Such twistors can be regarded as spinor Lorentz harmonics [10, 11]. Their only property to be essential for our purposes is that they can be used to define the appropriate vector moving frame. This allows one to avoid complicated calculations associated with the use of the spinor moving frame or spinor harmonic formalism [11 and to obtain the solution of nonlinear equations describing the extrinsic geometry of bosonic string moving in the Minkowski space of any dimension $D$ in terms of the vector Lorentz harmonics only.

Let us introduce two extra sets of Lorentz harmonics (moving frame variables)

$$
\begin{aligned}
& r_{\underline{\underline{a}}}^{(\underline{a})}\left(\xi^{(--)}\right)=\left(r_{\underline{\underline{m}}}^{( \pm \pm)}, r_{\underline{m}}^{i}\right) \quad \in \quad S O(1, D-1), \\
& l_{\underline{\underline{a}}}^{(\underline{a})}\left(\xi^{(++)}\right)=\left(l_{\underline{\underline{m}}}^{( \pm \pm)}, l_{\underline{m}}^{i}\right) \in S O(1, D-1),
\end{aligned}
$$

each depending only on the $\xi^{(--)}$and $\xi^{(++)}$coordinates of the string worldsheet.

Recall that the condition (78) means

$$
\begin{aligned}
r \frac{\underline{m}}{(\underline{a})} r_{\underline{m b}} & =\eta_{(\underline{a})(\underline{b})} \equiv \operatorname{diag}(1,-1, \ldots,-1), \\
\Leftrightarrow & \left\{\begin{array}{l}
r_{m}^{(++)} r \underline{m}(++)=0, \quad r_{\underline{m}}^{(--)} r \underline{m}(--)=0, \\
r_{\underline{m}}^{(++)} r \underline{m}(--)=2, \\
r_{\underline{m}}^{(++)} r \underline{m} i=0, \quad r_{\underline{m}}^{(--)} r \underline{m} i=0, \\
r_{\underline{m}}^{i} r \underline{m} j=-\delta^{i j} .
\end{array}\right. \\
l \underline{\underline{m}}(\underline{a}) \underline{\underline{m b}}= & \eta_{(\underline{a})(\underline{b})} \equiv \operatorname{diag}(1,-1, \ldots,-1), \\
\Leftrightarrow & \left\{\begin{array}{l}
l_{\underline{m}}^{(++)} l \underline{m}(++)=0, \quad l_{\underline{m}}^{(--)} l \underline{m}(--)=0, \\
l_{\underline{m}}^{(++)} l \underline{m}(--)=2, \\
l_{\underline{m}}^{(++)} l \underline{m} i=0, \\
l_{\underline{m}}^{i} l \underline{m} j=-\delta^{i j} .
\end{array}\right.
\end{aligned}
$$

Further, we identify the chiral vectors $\partial_{(--)} X \frac{m}{R}$ and $\partial_{(++)} X_{\frac{m}{L}}$ with the components $r^{(++)}=$ $r^{(++)}\left(\xi^{(--)}\right)$and $l^{(--)}=l^{(--)}\left(\xi^{(++)}\right)$of these sets of chiral harmonics

$$
\partial_{(++)} X_{L}^{\underline{m}}=\frac{1}{2} M_{(++)}^{(++)}\left(\xi^{(++)}\right) l^{(--)} \underline{m}\left(\xi^{(++)}\right), \quad \partial_{(--)} X_{R}^{\underline{m}}=\frac{1}{2} M_{(--)}^{(--)}\left(\xi^{(--)}\right) r^{(++)} \underline{m}\left(\xi^{(--)}\right) .
$$

In such a way we adapt the chiral frames to the left and right sectors of the string worldsheet. Since other components of the left- and right-moving frame variables $l_{\underline{\underline{a}}}^{(\underline{a})}, r_{\underline{\underline{a}}}^{(\underline{a})}$ remain arbitrary, we have just a "particle-like" situation in the present case. Hence, Eqs. (81) possess the invariance under the affine

$$
\left(S O(1,1) \otimes S O(D-2) \otimes K_{D-2}\right)_{L} \quad \text { and } \quad\left(S O(1,1) \otimes S O(D-2) \otimes K_{D-2}\right)_{L}
$$

symmetries with chiral parameters

$$
V_{L}\left(\xi^{(++)}\right)=e^{h_{L}}, \quad V_{R}^{(++) i}\left(\xi^{(++)}\right), \quad V^{i j}\left(\xi^{(++)}\right)=V^{-1 j i}\left(\xi^{(++)}\right)
$$


and

$$
V_{R}\left(\xi^{(--)}\right)=e^{h_{R}}, \quad V_{R}^{(--) i}\left(\xi^{(--)}\right), \quad V^{i j}\left(\xi^{(--)}\right)=V^{-1 j i}\left(\xi^{(--)}\right)
$$

respectively'. The corresponding transformations read

$$
\begin{aligned}
r_{\underline{m}}^{(++) \prime} & =r_{\underline{m}}^{(++)} V_{R}, \\
r_{\underline{m}}^{i \prime} & =\left(r_{\underline{m}}^{j}+r_{\underline{m}}^{(++)} V_{R}^{(--) i}\right) V_{R}^{j i}, \\
r_{\underline{m}}^{(--) \prime} & =\left(r_{\underline{m}}^{(--)}+r_{\underline{m}}^{(++)} V_{R}^{(--) i} V_{R}^{(--) i}+2 r_{\underline{m}}^{i} V_{R}^{--i}\right) V_{R}^{-1}, \\
l_{\underline{m}}^{(--)} & =l_{\underline{m}}^{(--)} V_{L}, \\
l_{\underline{m}}^{i} & =\left(l_{\underline{m}}^{j}+l_{\underline{m}}^{(--)} V_{L}^{(++) i}\right) V_{L}^{j i}, \\
l_{\underline{m}}^{(++)} & =\left(l_{\underline{m}}^{(++)}+l_{\underline{m}}^{(--)} V_{L}^{(++) i} V_{L}^{(++) i}+l_{\underline{m}}^{i} V_{L}^{(++) j}\right) V_{L}^{-1} .
\end{aligned}
$$

Hence, each set of chiral harmonics parametrizes the sphere (18). As they depend only on one of the worldsheet coordinates, $\xi^{(--)}$or $\xi^{(++)}$, they map one of the light-like sectors, $\mathcal{M}^{(0,1)}=\left\{\xi^{(--)}\right\}$or $\mathcal{M}^{(1,0)}=\left\{\xi^{(++)}\right\}$, of the worldsheet $\mathcal{M}^{(1,1)}=\left\{\xi^{m}\right\}=\left\{\xi^{(++)}, \xi^{(--)}\right\}$onto two copies of this sphere

$$
\begin{aligned}
& r_{\underline{\underline{a}}}^{(\underline{a})}: \quad \mathcal{M}^{(0,1)}=\left\{\xi^{(--)}\right\} \quad \rightarrow \quad S^{D-2}=\frac{S O(1, D-1)}{S O(1,1) \times S O(D-2) \otimes K_{D-2}}, \\
& l_{\underline{\underline{a}}}^{(\underline{a})}: \quad \mathcal{M}^{(1,0)}=\left\{\xi^{(++)}\right\} \quad \rightarrow \quad S^{D-2}=\frac{S O(1, D-1)}{S O(1,1) \times S O(D-2) \otimes K_{D-2}} .
\end{aligned}
$$

Below we denote the spaces of all possible images of these maps by $S_{L}^{(D-2)}$ and $S_{R}^{(D-2)}$, respectively.

The chiral counterparts of the Cartan forms (12) contain only one of the chiral holonomic basic 1-forms $d \xi^{(--)}$or $d \xi^{(++)}$

$$
\begin{aligned}
& f_{R}^{(++) i}=d \xi^{(--)} f_{(--) R}^{(++) i}=r^{(++) \underline{m}} d r_{\underline{m}}^{i}, \quad f_{(--) R}^{(++) i}=r^{(++) \underline{m}} \partial_{(--)} r_{\underline{m}}^{i} \\
& f_{R}^{(--) i}=d \xi^{(--)} f_{(--) R}^{(--) i}=r^{(--) \underline{m}} d r_{\underline{m}}^{i}, \quad f_{(--) R}^{(--) i}=r^{(--) \underline{m}} \partial_{(--)} r_{\underline{m}}^{i} \\
& \omega_{R}=d \xi^{(--)} \omega_{(--) R}=\frac{1}{2} r^{(--) \underline{m}} d r_{\underline{m}}^{(++)}, \quad \omega_{(--) R}=\frac{1}{2} r^{(--) \underline{m}} \partial_{(--)} r_{\underline{m}}^{(++)} \\
& A_{R}^{i j}=d \xi^{(--)} A_{(--) R}^{i j}=\frac{1}{2} r^{i \underline{m}} d r_{\underline{m}}^{j}, \quad A_{(--) R}^{i j}=\frac{1}{2} r^{i \underline{m}} \partial_{(--)} r_{\underline{m}}^{j} \\
& f_{L}^{(++) i}=d \xi^{(++)} f_{(++) L}^{(++) i}=l^{(++) \underline{m}} d l_{\underline{m}}^{i}, \quad f_{(++) L}^{(++) i}=l^{(++) \underline{m}} \partial_{(++)} l_{\underline{m}}^{i} \\
& f_{L}^{(--) i}=d \xi^{(++)} f_{(++) L}^{(--) i}=l^{(--) \underline{m}} d l_{\underline{m}}^{i}, \quad f_{(++) L}^{(--) i}=l^{(--) \underline{m}} \partial_{(++)} l_{\underline{m}}^{i}
\end{aligned}
$$

${ }^{4}$ The affine symmetry $S O(1,1)_{L} \otimes S O(1,1)_{R}$ proves to be "soldered" to the worldsheet conformal symmetry when the gauge $M_{(++)}^{(++)}=1=M_{(--)}^{(-)}$is imposed. 


$$
\begin{array}{ccc}
\omega_{L}=d \xi^{(++)} \omega_{(++) L}=\frac{1}{2} l^{(--)} \underline{m} d l_{\underline{m}}^{(++)}, & \omega_{(++) L}=\frac{1}{2} l^{(--) \underline{m}} \partial_{(++)} l_{\underline{m}}^{(++)} \\
A_{L}^{i j}=d \xi^{(++)} A_{(++) L}^{i j}=\frac{1}{2} l^{i \underline{m}} d l_{\underline{m}}^{j}, & A_{(++) L}^{i j}=\frac{1}{2} l^{i \underline{m}} \partial_{(++)} l_{\underline{m}}^{j}
\end{array}
$$

The transformations of these 1 -forms under the left and right affine $S O(1,1) \otimes S O(D-2) \subset$ $\times K_{D-2}$ symmetry (82) and (83) are determined by the chiral version of Eqs. (19)-(22) and its evident "left" counterpart respectively. It is worth noting that only the forms

$$
f_{R}^{(++) i}=d \xi^{(--)} f_{(--) R}^{(++) i} \quad \text { and } \quad f_{R}^{(--) i}=d \xi^{(++)} f_{(++) L}^{(--) i}
$$

transform covariantly under (82) and (83). These forms are vielbeins of the 'chiral spheres' $S_{R}^{D-2}$ (84) and $S_{R}^{D-2}$ (85), respectively.

\subsection{Relation of general and chiral harmonics: solving the Liouville-type equation}

Substituting Eq. (81) into Eq.(77), one obtains the expression for the chiral light-like moving frame vector fields $l^{(--) \underline{m}}\left(\xi^{(++)}\right), r^{(++)} \underline{m}\left(\xi^{(--)}\right)$in terms of generic light-like harmonics $u^{--\underline{m}}, u^{++\underline{m}}$, the Liouville field $W$ and compensator $L$

$$
l^{(--) \underline{m}}\left(\xi^{(++)}\right)=e^{-W-L} u^{-\underline{m}}, \quad r^{(++) \underline{m}}\left(\xi^{(--)}\right)=e^{-W+L} u^{++\underline{m}} .
$$

Contracting both sides of these two equations in indices $\underline{m}$, we get the expression for the field $W$ in terms of chiral harmonics

$$
e^{-2 W}=\frac{1}{2} l^{(--)} \underline{m} r_{\underline{m}}^{(++)} .
$$

For $D=3$ Eq. (95) produces the general solution of the Liouville equation in a special parametrization (see Appendix ).

A similar solution for the Liouville equation in the conformal gauge was presented in Ref. [8]. The Cartan-Penrose representation in terms of bosonic spinors was used there for chiral light-like vectors $l^{(--)} \underline{m}$ and $r_{m}^{(++)}$.

In the generic case of higher $D$ it is necessary to get the suitable representation for the $S O(D-2)$ matrices $G^{i j}$ as well.

\subsection{Relation of general and chiral harmonics: solving the sigma-model-type equation}

To obtain the expression for $S O(D-2)$ matrix field $G$, let us analyze the consequences of Eq. (94) for the moving frame vectors $u^{i}$. First of all, one finds

$$
l^{(--) \underline{m}} u_{\underline{m}}^{i}=0, \quad r^{(++) \underline{m}} u_{\underline{m}}^{i}=0
$$

Eqs. (96) mean that the decompositions of the $u^{i}$ harmonic over the chiral left- and right-moving ones have no terms proportional to $l^{(++)}$and $r^{(--)}$, respectively

$$
u_{\underline{m}}^{i}=-\left(l_{\underline{m}}^{j}-V_{(--)}^{j} l_{\underline{m}}^{(--)}\right) U_{\mathcal{L}}^{j i}
$$




$$
u_{\underline{m}}^{i}=-\left(r_{\underline{m}}^{j}+V_{(++)}^{i} \underline{r_{m}}(++)\right) U_{\mathcal{R}}^{j i} .
$$

The newly introduced matrices $U_{\mathcal{L}}^{j i}$ and $U_{\mathcal{L}}^{j i}$ are expressed through the contractions of chiral harmonics with the generic ones as follows

$$
U_{\mathcal{L}}^{j i}=l^{j \underline{m}} u_{\underline{m}}^{i}, \quad U_{\mathcal{R}}^{j i}=r^{j \underline{m}} u_{\underline{m}}^{i} .
$$

So they can easily be checked to be orthogonal matrices

$$
U_{\mathcal{L}}^{j k} U_{\mathcal{L}}^{i k}=\delta^{j i}, \quad U_{\mathcal{L}}^{j k} U_{\mathcal{L}}^{i k}=\delta^{j i} .
$$

The proof uses the unity decomposition (7) and the corollaries of Eqs. (94)

$$
u^{-\underline{\underline{m}}} l_{\underline{m}}^{i}=0, \quad u^{++\underline{m}} r_{\underline{m}}^{i}=0 .
$$

To find the relations between $U_{\mathcal{L}, \mathcal{R}}$ and $G_{\mathcal{R}, \mathcal{L}}$, one should consider the derivatives of the $U_{\mathcal{L}, \mathcal{R}}$ fields

$$
\partial_{(--)} U_{\mathcal{L}}^{j i}=l^{j \underline{m}} \partial_{(--)} u_{\underline{m}}^{i} \quad \text { and } \quad \partial_{(++)} U_{\mathcal{R}}^{j i}=r^{j \underline{m}} \partial_{(++)} u_{\underline{m}}^{i}
$$

and use the decomposition (11) to express the derivatives of the generic harmonics in terms of components of the Cartan forms (51)-(50). In such a way one arrives at

$$
\left(\partial_{(--)} U_{\mathcal{L}}^{k j}\right) U_{\mathcal{L}}^{k i}=\left(\partial_{(--)} G_{\mathcal{R}}^{j k}\right) G_{\mathcal{R}}^{i k}, \quad\left(\partial_{(++)} U_{\mathcal{R}}^{k j}\right) U_{\mathcal{R}}^{k i}=\left(\partial_{(++)} G_{\mathcal{L}}^{j k}\right) G_{\mathcal{L}}^{i k}
$$

Eqs. (102) mean that the $G_{\mathcal{L}}\left(G_{\mathcal{R}}\right)$ field differs from the (transposed) matrix field $U_{\mathcal{R}}\left(U_{\mathcal{L}}\right)$ by an affine $S O(D-2)_{L}\left(S O(D-2)_{R}\right)$ transformation only (cf. (70))

$$
\begin{gathered}
G_{\mathcal{R}}^{i j}=H_{L}^{j k}\left(\xi^{(++)}\right) U_{\mathcal{L}}^{k i}=u^{i \underline{m}} l_{\underline{m}}^{k} H_{L}^{k j}\left(\xi^{(++)}\right), \\
G_{\mathcal{L}}^{i j}=H_{R}^{j k}\left(\xi^{(--)}\right) U_{\mathcal{R}}^{k i}=u^{i \underline{m}} r_{\underline{m}}^{k} H_{R}^{j k}\left(\xi^{(-)}\right), \\
H_{L} H_{L}^{T}=I, \quad \partial_{(--)} H_{L}=0, \quad H_{R} H_{R}^{T}=I, \quad \partial_{(++)} H_{R}=0 .
\end{gathered}
$$

The expression for $V_{(++)}^{i}$ follows from the first equation in (96) upon substituting (98) and using Eqs.(94), (103), (95). In this way one gets

$$
V_{(++)}^{i}=\frac{1}{2} e^{2 W} l^{(--)} \underline{m} r_{\underline{m}}^{i} .
$$

In the same manner one can obtain

$$
V_{(--)}^{i}=\frac{1}{2} e^{2 W} r^{(++) \underline{m}} l_{\underline{m}}^{i}
$$

from Eq. (97) and the second of Eqs. (96).

Now we can rewrite Eqs. (97), (98) in terms of chiral harmonics and the functions entering the nonlinear equations (53), (54)

$$
\begin{gathered}
u_{\underline{m}}^{i}=G_{\mathcal{R}}^{i k} H_{L}^{k j}\left(-l_{\underline{m}}^{j}+e^{2 W} r^{(++)}{ }_{\underline{n}}^{j} l_{\underline{m}}^{j} l^{(--)}\right), \\
u_{\underline{m}}^{i}=G_{\mathcal{L}}^{i k} H_{R}^{k j}\left(-r_{\underline{m}}^{j}+e^{2 W} l^{(--)} \underline{n} r_{\underline{\underline{n}}}^{j} r_{\underline{m}}^{(++)}\right) .
\end{gathered}
$$


From Eqs. (107) and (108) we obtain the expression

$$
\left(G_{\mathcal{L}}^{T} G_{\mathcal{R}}\right)^{i j}=H_{L}^{i k}\left(-l^{k \underline{m}} r_{\underline{m}}^{l}+\frac{2\left(r^{(++)} \underline{\underline{n}} l_{\underline{\underline{m}}}^{k}\right)\left(l^{(--)} \underline{n^{\prime}} r_{\underline{\underline{n}^{\prime}}}^{l}\right)}{\left(l^{(--) \underline{\underline{n}^{\prime \prime}}} r_{\underline{n^{\prime \prime}}}^{(++)}\right)}\right) H_{R}^{j l},
$$

which provides the general solution for the sigma-model-like equation (1) in the gauge (67)

$$
\begin{gathered}
G_{\mathcal{R}}^{i j}=\delta^{i j}, \quad G_{\mathcal{L}}^{i j} \equiv G^{i j}, \\
G^{i j}=H_{L}^{i k}\left(-l^{k \underline{m}} r_{\underline{m}}^{l}+\frac{2\left(r^{(++)} l_{\underline{n}}^{k}\right)\left(l^{(--) \underline{n^{\prime}}} r_{\underline{n}^{\prime}}^{l}\right)}{\left(l^{(--)} \underline{\underline{n}^{\prime \prime}} r_{\underline{n^{\prime \prime}}}^{(++)}\right)}\right) H_{R}^{j l} .
\end{gathered}
$$

To complete the description of the general solution of the string-inspired system of nonlinear equations (2), (11), we have to present the expressions for chiral vector fields $M_{( \pm \pm)}^{(\mp \mp) i}$ (3) in terms of the chiral harmonics. This can be easily done by applying the derivatives $\partial_{(++)}$and $\partial_{(--)}$ to both sides of Eqs. (107), (108) and contracting the results with the vectors $l^{(++)}$and $r^{(--)}$, respectively. Then, using Eqs. (51), (52), one obtains

$$
\begin{gathered}
M_{(--)}^{(++) i}=-H_{R}^{i j} r^{(++) \underline{m}} \partial_{(--)} r_{\underline{m}}^{j}, \\
M_{(++)}^{(--) i}=-H_{L}^{i j} l^{(--) \underline{m}} \partial_{(++)} l_{\underline{m}}^{j} .
\end{gathered}
$$

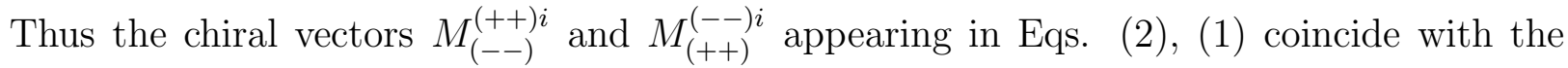
covariant components $f_{( \pm \pm)}^{(\mp \mp) i}$ (86), (91) of the chiral Cartan forms which form a basis on the "chiral spheres" $S_{R}^{D-2}$ and $S_{L}^{D-2}$, respectively.

\subsubsection{General solution of the string-inspired nonlinear equations}

Eqs. (95), (110), (111) and (112) provide the general solution for the system of nonlinear equations (2), (11), (3).

To be convinced of this, one has to take into account the following.

- When obtaining the expressions (95), (110), (111), (112) for all the functions which enter Eqs. (2), (1), (3), we started from the general solution of the bosonic string equations of motion in the standard Nambu-Goto approach and then used these solutions in the equations of the geometric approach.

- As we demonstrated in Section 1, the equations of the geometric approach describing the extrinsic geometry of the D-dimensional bosonic string worldsheet uniquely produce the system of nonlinear equations (21), (68), (3).

- The equations of geometric approach and, therefore, Eqs. (22), (11), (33) specify the bosonic string worldsheet uniquely (up to symmetry transformations, see e.g. [4]), and thus describe exactly the same dynamical system as the ordinary (linear) string equations of motion.

- The general solution (95), (110), (111), (112) is written in terms of two sets of chiral spinor harmonics (78), which parametrize two copies of the compact coset (18)

$$
S^{D-2}=\frac{S O(1, D-1)}{S O(1,1) \times S O(D-2) \otimes K_{D-2}}
$$


isomorphic to the sphere $S^{D-2}$. Thus it contains $(D-2)$ right-moving and $(D-2)$ leftmoving degrees of freedom, the same number as that of independent degrees of freedom of the general solution (74) of the standard string equations of motion (71).

With this reasoning in mind, we conclude that the expressions (95), (110), (111) and (112) for the functions $W, G$ and $M_{(++)}^{(-) i}, M_{(--)}^{(++) i}$ obtained from the general solution of the standard string equations have to provide the general solution of the geometric approach equations Eqs. (21), (11), (3) (up to superfluous symmetry transformations).

\section{On the group theoretical and geometrical structure of the solution}

In the course of deriving the general solution (95), (110), (111), (112) we have got the expressions for the moving frame vectors (44) in terms of chiral harmonics

$$
\begin{aligned}
u_{\underline{m}}^{++} & =e^{W-L} r_{\underline{m}}^{(++)}, \\
u_{\underline{m}}^{i} & =-\left(G_{\mathcal{L}} H_{R}\right)^{i j}\left(r_{\underline{m}}^{j}-V_{(++)}^{j} r_{\underline{m}}^{(++)}\right), \\
u_{\underline{m}}^{--} & =e^{-(W-L)}\left(r_{\underline{m}}^{(--)}+r_{\underline{m}}^{(++)} V_{(++)}^{j} V_{(++)}^{j}-2 r_{\underline{m}}^{i} V_{(++)}^{i}\right), \\
u_{\underline{m}}^{++} & =e^{-(W+L)}\left(l_{\underline{m}}^{(++)}+l_{\underline{m}}^{(--)} V_{(--)}^{j} V_{(--)}^{j}-2 l_{\underline{m}}^{i} V_{(--)}^{i}\right), \\
u_{\underline{m}}^{i} & =-\left(G_{\mathcal{R}} H_{L}\right)^{i j}\left(l_{\underline{m}}^{j}-V_{(--)}^{j} r_{\underline{m}}^{(--)}\right), \\
u_{\underline{m}}^{--} & =e^{W+L} r_{\underline{m}}^{(-)} .
\end{aligned}
$$

If the functions $W$ and $G_{\mathcal{L}, \mathcal{R}}$ satisfy the nonlinear equations (2), (11), then Eqs. (113), (114) can be regarded as the solution of the corresponding associated linear system defined by Eqs. (11]), with the Cartan forms (12) being specified by Eqs. (51), (52), (49), (501)).

The solution of the zero curvature representation given by the Maurer-Cartan equations (34) with the Cartan forms (12) can be obtained by differentiating (113), (114). The solution is presented by the following expressions for the generic Cartan 1-forms (13)-(16) in terms of chiral once (86)-(89) and (90)-(93):

$$
\begin{gathered}
f^{++i}=e^{W-L}\left(G_{\mathcal{L}} H_{R}\right)^{i j} f_{R}^{(++) j}, \\
f^{--i}=-e^{-(W-L)}\left(G_{\mathcal{L}} H_{R}\right)^{i j}\left(f_{R}^{(--) j}-2 \mathcal{D}_{R} V_{(++)}^{j}-2 f_{R}^{(++) k}\left(V_{(++)}^{k} V_{(++)}^{j}-\frac{1}{2} \delta^{k j} V_{(++)} V_{(++)}{ }^{l}\right)\right), \\
\mathcal{D}_{R} V_{(++)}^{j} \equiv d V_{(++)}^{j}+\omega_{R} V_{(++)}{ }^{j}-V_{(++)}{ }^{k} A_{R}^{k j} \\
\omega=\omega_{R}+f^{(++) i} V_{(++)}^{i}+d(W-L), \\
\left.A^{i j}=\left(G_{\mathcal{L}} H_{R}\right)^{i k}\left(G_{\mathcal{L}} H_{R}\right)^{j l}\left(A_{R}^{k l}+\left(G_{\mathcal{L}} H_{R}\right)^{-1} d\left(G_{\mathcal{L}} H_{R}\right)\right)^{k l}+2 f^{(++)[k} V^{--l]}\right),
\end{gathered}
$$

\footnotetext{
${ }^{5}$ More precisely, we have got the first two equations in each set (113), (114) while the third one can be restored using the orthonormality conditions (5), (79), (80).
} 


$$
\begin{gathered}
f^{--i}=e^{W+L}\left(G_{\mathcal{R}} H_{L}\right)^{i j} f_{L}^{(--) j} \\
f^{++i}=-e^{-(W-L)}\left(G_{\mathcal{R}} H_{L}\right)^{i j}\left(f_{L}^{(++) j}-2 \mathcal{D}_{R} V_{(--)}^{j}-2 f_{L}^{(--) k}\left(V_{(--)} V_{(--)}^{j}-\frac{1}{2} \delta^{k j} V_{(--)} V_{(--)}\right)\right) \\
\mathcal{D}_{L} V_{(--)}^{j} \equiv d V_{(--)}^{j}+\omega_{R} V_{(--)}^{j}-V_{(--)} A_{L}^{k j} \\
\omega=\omega_{R}+f^{(--) i} V_{(--)}{ }^{i}-d\left(W^{j} L\right) \\
\left.A^{i j}=\left(G_{\mathcal{L}} H_{R}\right)^{i k}\left(G_{\mathcal{L}} H_{R}\right)^{j l}\left(A_{R}^{k l}+\left(G_{\mathcal{L}} H_{R}\right)^{-1} d\left(G_{\mathcal{L}} H_{R}\right)\right)^{k l}+2 f^{++[k} V^{--l]}\right)
\end{gathered}
$$

On the other hand, as follows from the consideration in the previous section, the explicit form of the general solution (95), (110), as well as the expressions (105), (106) for the 'boost' parameters, can be obtained algebraically from Eqs. (114), (113) (with making use of the orthonormality constraints (5), (79), (80) for the generic and chiral moving frame harmonics).

An intriguing point is that Eqs. (114), (113) generating the general solution have the form of the parabolic symmetry transformations (83), (82) of the chiral harmonics, but with non-chiral parameters.

Thus the prescription of how to solve the nonlinear equations (11), (2) can be formulated as follows.

Let us introduce the two set of chiral harmonics $(78),(79),(79)$ which define maps of the right (left) light-cone sectors of the worldsheet $\mathcal{M}^{(0,1)} \equiv\left\{\left(\xi^{(--)}\right)\right\}\left(\mathcal{M}^{(1,0)} \equiv\left\{\left(\xi^{(++)}\right)\right\}\right)$onto the sphere $S^{D-2}$

$$
\begin{aligned}
& r_{\underline{\underline{a}}}^{(\underline{a})}: \mathcal{M}^{(0,1)} \equiv\left\{\left(\xi^{(--)}\right)\right\} \quad \rightarrow \quad S^{D-2}=\frac{S O(1, D-1)}{S O(1,1) \times S O(D-2) \otimes K_{D-2}}, \\
& r_{\underline{\underline{a}}}^{(\underline{a})}: \quad \mathcal{M}^{(1,0)} \equiv\left\{\left(\xi^{(++)}\right)\right\} \quad \rightarrow \quad S^{D-2}=\frac{S O(1, D-1)}{S O(1,1) \times S O(D-2) \otimes K_{D-2}} .
\end{aligned}
$$

Further, let us assume that the generic harmonics (4), (5)

$$
u_{\underline{\underline{m}}}^{\underline{a}}: \mathcal{M}^{(1,1)} \rightarrow \frac{S O(1, D-1)}{S O(1,1) \times S O(D-2)}
$$

are related to the chiral ones by the parabolic transformations (113), (114) (with the chiral parameters $H_{L}$ and $H_{R}$ omitted for simplicity).

Then let us exploit the $S O(D-2)$ gauge freedom to fix the $S O(D-2)$ rotation matrix in (113) to be the unity one. The $S O(D-2)$ rotation matrix in (114) taken in this gauge provides us with the solution (110) of the WZNW sigma-model-type equation (1) (with the chiral vectors $M_{(--)}^{(+) i}, M_{(--)}^{(++) i}$ determined by the homogeneously transforming components of chiral Cartan forms (86), (91)

$$
M_{(--)}^{(++) i}=f_{(--) R}^{(++) i}, \quad M_{(--)}^{(++) i}=f_{(++) L}^{(--) i}
$$

(cf. (112), (111))). The product of the $S O(1,1)$ transformation factors from (113) and (113) produces the general solution of the Liouville-like equation (2). 
Since the parabolic transformations (114), (113) have a "triangular" form, one can expect that the described method of getting the general solution bears a tight relation to the known group-theoretical methods of solving nonlinear equations, like those developed and used in [14, 15, 16]. A detailed examination of such a relationship could provide a deeper insight into the nature of integrability and we consider it as an interesting problem for the future study.

\section{Conclusion}

Thus we have obtained the general solution of the string-inspired nonlinear equations (1), (53), (3) describing the extrinsic geometry of the bosonic string worldsheet in the geometrical approach [1, 2, 3, 5].

The solution is given in terms of the two sets of chiral (left-moving and right-moving) Lorentz harmonic variables $(78),(79),(80)$ and has the form (after fixing a gauge with respect to some extra symmetries)

$$
\begin{gathered}
e^{-2 W}=\frac{1}{2} r_{\underline{m}}^{(++)} l^{(--) M}, \\
G^{k j}=-l_{\underline{m}}^{k} r^{j \underline{m}}+\frac{r_{\underline{m}}^{(++)} l^{k \underline{m}} l_{\underline{n}}^{(--)} r^{j \underline{n}}}{r_{\underline{p}}^{(++)} l^{(--)} \underline{p}}, \\
M_{(--)}^{(++) i}\left(\xi^{(--)}\right)=r^{(++) \underline{m}} \partial_{(--)} r_{\underline{m}}^{i}, \\
M_{(++)}^{(--) i}\left(\xi^{(++)}\right)=l^{(--) \underline{m}} \partial_{(++)} l_{\underline{m}}^{i} .
\end{gathered}
$$

The analysis of the solution of the associated linear system demonstrates that the general solution we have found can be regarded as the parabolic subgroup $S O(1,1) \times S O(D-2) \otimes K_{D-2}$ transformations [10] of the chiral harmonics with non-chiral parameters. As these transformations are of a "triangle" form in the matrix representation, we can expect a close relation of our approach to the existing group-theoretical methods of solving nonlinear equations [14, 15, 16]. It is an interesting task for further study to elaborate such a relation in more detail.

A natural direction of extending our results is to look for the solution of a supersymmetric generalization of the considered nonlinear equations. Such a system describes the extrinsic geometry of the worldsheet superspace of $D=3,4,6,10$ superstring models 0 .

\section{Acknowledgments}

The authors are grateful to D. Sorokin, S. Krivonos, B. Julia, F. Toppan for interest to this work and useful remarks. One of the author (I.B.) would like to thank Prof. M. Virasoro and Prof. S. Randjbar-Daemi for the hospitality at the Abdus Salam ICTP, where the part of this work was done. The work was supported in part by the INTAS Grants 96-0308, 96-0538, 93127ext, the RFBR Grant 96-02-17634 and by the Fonds zur Förderung der wissenschaftlichen Forschung under Project No M472-TPH (IB).

\footnotetext{
${ }^{6}$ Let us note that the explicit form of such supersymmetric equations are known at present for the $D=$ $3, N=1,2$ cases [17] only.
} 


\section{Appendix : Relation with the standard form of general solution of the Liouville equation}

Here we demonstrate that for $D=3$ case Eq. (95) reproduces the general solution of the nonlinear Liouville equation. Using the well-known parametrization of the $S O(1,2)$ matrices

$$
\begin{aligned}
l \underline{\underline{a}})= & \left(\begin{array}{ccc}
\cosh A_{L} & \sinh A_{L} & 0 \\
\sinh A_{L} & \cosh A_{L} & 0 \\
0 & 0 & 1
\end{array}\right), \\
r \frac{m}{(\underline{a})}= & \left(\begin{array}{ccc}
\cosh A_{R} & -\sinh A_{R} & 0 \\
-\sinh A_{R} & \cosh A_{R} & 0 \\
0 & 0 & 1
\end{array}\right), \\
& \partial_{(--)} A_{L}=\partial_{(++)} A_{R}=0
\end{aligned}
$$

one finds the following form of chiral harmonics (78)

$$
\begin{aligned}
l^{(--) \underline{m}} & =\left(\begin{array}{lll}
\cosh A_{L}, & \sinh A_{L}, & 1
\end{array}\right), \\
l^{\perp \underline{m}} & =\left(\begin{array}{lll}
\sinh A_{L}, & \cosh A_{L}, & 0
\end{array}\right), \\
l^{(++) \underline{m}} & =\left(\begin{array}{lll}
\cosh A_{L}, & \sinh A_{L}, & -1
\end{array}\right), \\
r^{(--) \underline{m}} & =\left(\begin{array}{lll}
\cosh A_{R}, & -\sinh A_{R}, & 1
\end{array}\right), \\
r^{\perp \underline{m}} & =\left(\begin{array}{lll}
-\sinh A_{R}, & \cosh A_{R}, & 0
\end{array}\right), \\
r^{(++) \underline{m}} & =\left(\begin{array}{lll}
\cosh A_{R}, & -\sinh A_{R}, & -1
\end{array}\right) .
\end{aligned}
$$

Substituting these expressions into the Eqs. (95), (111), (112) with $D=3$, one gets

$$
\begin{gathered}
e^{-W}=\cosh \frac{A_{L}+A_{R}}{2}, \\
M_{(--)}^{++\perp}\left(\xi^{(--)}\right)=r^{(++) \underline{m}} \partial_{(--)} r_{\underline{m}}^{\perp}=-\partial_{(--)} A_{R}, \\
M_{(++)}^{--\perp}\left(\xi^{(++)}\right)=l^{(--) \underline{m}} \partial_{(++)} l_{\underline{m}}^{\perp}=\partial_{(++)} A_{L} .
\end{gathered}
$$

The relation to the standard parametrization of the general solution of the Liouville equation (see e.g. [18]) is given by

$$
f_{L, R}=\exp A_{L, R}
$$

\section{References}

[1] F. Lund and T. Regge, Phys.Rev. D14 (1976) 1524;

R. Omnes, Nucl. Phys. B 149 ((1979)) 269;

B.M. Barbashov and V.V. Nesterenko, Commun.Math.Phys. 78 (1981) 499.

A. Zheltukhin, Sov.J.Nucl.Phys. 33 (1981) 1723; Theor.Math.Phys. 52 (1982) 73; Phys.Lett. B116 (1982) 147; Theor.Math.Phys. 56 (1983) 230.

[2] B.M. Barbashov and V.V. Nesterenko, "Introduction to the relativistic string theory", World Scientific, 1990 (and refs.therein). 
[3] I.A. Bandos, P. Pasti, D. Sorokin, M. Tonin and D.V. Volkov, Nucl. Phys. B 446 (1995) 79.

[4] L.P. Eisenhart, "Riemannian geometry", Princeton Univ. Press, 1949.

[5] I.A. Bandos, Phys. Lett. B 388 (1996) 35.

[6] A. Galperin, E. Ivanov, S. Kalitzin, V. Ogievetsky and E. Sokatchev, Class. Quantum Grav. 1 (1984) 498; Class. Quantum Grav. 2 (1985) 155.

[7] E. Sokatchev, Phys.Lett. B169 (1987) 209; Class. Quantum Grav. 4 (1987) 237.

[8] A.A. Kapustnikov and S.A. Ulanov, Constrained twistors principle of the string theory, hepth/9704068.

[9] I.A. Bandos, Sov.J.Nucl.Phys. 51 (1990) 906; Sov. Phys. JETP Lett. 52 (1990) 205.

[10] A. Galperin, P. Howe and K. Stelle, Nucl. Phys. B 368 (1992) 248;

A. Galperin, F. Delduc and E. Sokatchev, Nucl. Phys. B 368 (1992) 143;

A. Galperin, K. Stelle and P. Townsend, Nucl. Phys. B 402 (1993) 531.

[11] I.A. Bandos and A.A. Zheltukhin, Sov. Phys. JETP Lett. 54 (1991) 421; Phys. Lett. B 288 (1992) 77; Sov.J.Nucl.Phys. 56 (1993) 198; Phys. Part. Nucl. 25 (1994) N5. P.453-477 [10651127]; Class. Quantum Grav. 12 (1995) 609.

[12] M. Green, J. Schwarz and E. Witten, Superstring theory. V.1,2, CUP 1987.

[13] L.D. Faddeev and L.A. Takhtajan, Hamiltonian Approach in Theory of Solitons, Springer, Berlin 1987.

[14] A.N. Leznov, M.V. Savelev, Commun.Math.Phys. 74 (1980) 111;

A.N. Leznov, Theor.Math.Phys. 73 (1987) 1233;

A.N. Leznov, M.A. Mukhtarov, J.Math.Phys. 28 (1987) 2574;

Ch. Devchand and A.N. Leznov, Commun.Math.Phys. 160 (1994) 551 (and refs. therein).

[15] E.A. Ivanov and S.O. Krivonos, Theor.Math.Phys. 58 (1984) 131 [200];

E.A. Ivanov and S.O. Krivonos, Theor.Math.Phys. 66 (1984) 60 [90];

E.A. Ivanov, S.O. Krivonos, V.M. Leviant, Nucl.Phys. B304 (1988) 601.

[16] E.A. Ivanov, Theor.Math.Phys. 71 (1987) 193;

E.A. Ivanov, $N=4$ supersymmetric integrable systems hep-th/9704133 (and refs. therein).

[17] I. Bandos, D. Sorokin and D.V. Volkov, Phys.Lett. B372 (1996) 77.

[18] M. Chaichian and P. P. Kulish, Phys. Lett. B 78 (1978) 413. 